\title{
OCORRÊNCIA DE CASOS DE DOENÇAS DIARREICAS AGUDAS E SUA RELAÇÃO COM OS ASPECTOS SANITÁRIOS NA REGIÃO DO ALTO TIETE, SÃO PAULO
}

\section{OCCURRENCE OF CASES OF ACUTE DIARRHIC DISEASES AND THEIR RELATIONSHIP WITH HEALTH ASPECTS IN THE ALTO TIETE REGION, SÃO PAULO}

\author{
Regina de Oliveira Moraes Arruda \\ Engenheira Agrônoma - Doutora em Tecnologia das Fermentações - FCF - USP \\ Mestrado em Análise Geoambiental - Universidade UNIVERITAS UNG \\ rarruda@prof.ung.br \\ Pedro Carmo de Souza \\ Biólogo -Mestre em Análise Geoambiental - Universidade UNIVERITAS UNG \\ Coordenadoria de Controle de Doenças (CCD), Secretaria de Estado da Saúde-SP \\ biopedrosouza@gmail.com \\ Edna Ferreira Rosini \\ Bióloga - Doutora em Biodiversidade Vegetal e Meio Ambiente- IB \\ Mestrado em Análise Geoambienta I- Universidade UNIVERITAS UNG \\ edna.ferreira@prof.ung.br \\ Fernanda Dall'Ara Azevedo \\ Bióloga - Doutora em Ecologia - UFRJ \\ Universidade UNIVERITAS UNG \\ fdallara.azevedo@gmail.com
}

\begin{abstract}
RESUMO
A falta de saneamento e de acesso às redes de captação e coleta de esgoto são fatores cruciais na veiculação de doenças principalmente as parasitárias que estão relacionadas com excretas capazes de poluir o ambiente e interferir na saúde, sendo os principais sintomas a diarreia. O objetivo da pesquisa foi realizar o levantamento das notificações de ocorrências de casos de doenças diarreicas agudas, no período de 2011 e 2015, nos onze municípios pertencentes ao Grupo de Vigilância Epidemiológica - VIII de Mogi das Cruzes$\mathrm{SP}$ e relacioná-los com indicadores de saneamento. As informações para a pesquisa foram obtidas a partir do Sistema Informatizado de Vigilância Epidemiológico do Ministério da Saúde disponibilizado pelo Centro de Vigilância Epidemiológica de São Paulo, nos relatórios anuais da Companhia Ambiental do Estado de São Paulo, e na Secretaria Nacional de Saneamento Ambiental do Ministério das Cidades. Com relação à doenças diarreicas agudas observou-se que houve relação entre as notificações e população atendida com água tratada, população atendida com esgoto e população rural dos municípios. Não se observou relação entre notificações e o Índice de Coleta e Tratabilidade de Esgotos Municipal. Os serviços em saneamento são fundamentais para promoção da saúde, neste caso, a redução das doenças diarreicas agudas.
\end{abstract}

Palavras chave Saneamento. Diarreia. Qualidade da água. Esgotos.

\section{ABSTRACT}

The lack of sanitation and access to sewage captation and collection networks are crucial factors in the transmission of diseases, especially parasitic diseases that are related to excreta also being able to pollute the environment and interfere with health, with the main symptom being diarrhea. The objective of the research was to survey the occurrence of reports of cases of Acute Diarrheal Diseases in the eleven municipalities belonging to the Epidemiological Surveillance Group - VIII of Mogi das Cruzes, and relate them to sanitation indicators, from 2011 to 2015. Information for the research was obtained from the Computerized Epidemiological Surveillance System of the Ministry of Health provided by the Center for Epidemiological Surveillance of São Paulo, also in the annual reports of the
\end{abstract}

Recebido em: 03/08/2019

Aceito para publicação em: 04/03/2020 
Environmental Company of the State of São Paulo, and the National Secretariat of Environmental Sanitation of the Ministry. of the cities. Regarding Acute Diarrheal Diseases, it was observed that there was a relationship between the notifications and population served with treated water, population served with sewage and rural population of the municipalities. There was no relationship between notifications and collection rate and sewage treatability. Sanitation services are fundamental for health promotion, in this case, the reduction of acute diarrheal diseases.

Key words: Sanitation. Diarrhea. Water quality. Sewers.

\section{INTRODUÇÃO}

O crescimento urbano desordenado e a falta de infraestrutura têm levado a piora da qualidade dos recursos hídricos o que pode gerar um problema de saúde pública, como as doenças de veiculação hídrica causadas por bactérias, vírus, protozoários intestinais e helmintos como febre tifoide, Hepatite tipo C, amebíase, ascaridíase entre outras (BRAGA, 2003).

As doenças diarreicas estão entre as principais causas de morbimortalidade principalmente em crianças e jovens nos países em desenvolvimento. É um grave problema de saúde pública e está intrinsicamente relacionado com as condições de higiene e água imprópria para o consumo (PAZ; ALMEIDA; GÜNTHER, 2012). Estas doenças acometem a população em geral, independente da faixa etária, porém, como indica a Organização Mundial de Saúde (OMS) (WHO, 2005), a maior ocorrência tem sido apontada em crianças menores de cinco anos de idade, sendo que cerca de um milhão e meio delas morrem de complicações relacionadas a doenças diarreicas a cada ano, nos países subdesenvolvidos.

Em São Paulo, temos o Indicador de Coleta e Tratabilidade de Esgotos Municipais - ICTEM, criado para verificar a situação dos municípios paulistas quanto ao desempenho de seus sistemas de coleta e tratamento de esgoto. O ICTEM tem por objetivo verificar a efetiva remoção da carga orgânica poluidora em relação à carga orgânica potencial gerada pelas populações urbanas municipais. Neste índice outros aspectos são englobados, além da coleta e o tratamento dos esgotos, a destinação dada aos lodos gerados nas estações (ETE), bem como os impactos causados aos corpos hídricos receptores dos efluentes (CETESB, 2018). Miranda, Braga (2011) usaram o ICTEM e o índice de Qualidade de Aterro de Resíduos - IQR para compor um İndice de Desempenho na Gestão a fim de auxiliar no processo de avaliação das políticas públicas e à tomada de decisões em relação a melhora do saneamento básico nos municípios.

Entre o período de 2007 a 2015, foram registrados, no Brasil, 34.568.644 casos de Doenças Diarreicas Agudas DDA sendo a maioria provocada por água contaminada (BRASIL, 2018). Esta alta incidência das doenças diarreicas demonstra a falta de infraestrutura urbana como a canalização dos córregos, captação e tratamento de esgoto, coleta e destinação do lixo, assim como tantos outros fatores que permitem a exposição de pessoas a riscos ambientais e consequentemente a doenças (JACOBI, 1995).

Por ser um sensível indicador de saúde pública das mais variadas alterações e mudanças nas condições de saneamento na qualidade sanitária de alimentos, hábitos de higiene ou mesmo diante a mudança de comportamento de uma população, o Ministério da Saúde instituiu desde o ano de 1994 o programa de Monitoramento das Doenças Diarreicas Agudas (MDDA) com o objetivo de detectar e monitorar nos municípios a incidência das diarreias. (QUEIROZ et al., 2009).

Os Grupos de Vigilância Epidemiológica (GVE) são regionalizados e regidos pela Coordenadoria de Controle de Doenças (CCD) e em suas áreas de abrangência tem como atribuição dar apoio técnico aos Departamentos de Vigilâncias Epidemiológicas municipais através de supervisão e coordenação das ações e medidas preventivas de fatores de doenças ou agravos. São trinta e um GVE estrategicamente regionalizados, sendo que o Grupo de Vigilância Epidemiológica - GVE-VIII de Mogi das Cruzes tem como abrangência os municípios de Arujá, Biritiba - Mirim, Ferraz de Vasconcelos, Guararema, Guarulhos, Itaquaquecetuba, Mogi das Cruzes, Poá, Salesópolis, Santa Isabel e Suzano 
(BRASIL, 2005), pertencentes a Região Metropolitana de São Paulo, denominada região do Alto Tietê.

Assim, dada a importância das doenças diarreicas para saúde pública,o objetivo desta pesquisa foi realizar o levantamento da ocorrência dos casos de doenças diarreicas agudas na Região do Alto Tietê, no período de 2011 a 2015, através dos dados obtidos do Sistema de Monitoramento do SIVEP- DDA do Ministério da Saúde disponibilizado pelo Centro de Vigilância Epidemiológica- CVESão Paulo e relacioná-los com indicadores de saneamento.

\section{MÉTODOS}

A análise da ocorrência de casos de doenças diarreicas na região do Alto Tiete foi realizada em 11 municípios pertencentes a zona Leste da Região Metropolitana de São: Paulo, Arujá, Biritiba - Mirim, Ferraz de Vasconcelos, Guararema, Guarulhos, Itaquaquecetuba, Mogi das Cruzes, Poá, Salesópolis, Santa Isabel e Suzano, todos pertencentes a área de abrangência do Grupo de Vigilância Epidemiológica - GVE-VIII de Mogi das Cruzes-SP..

Os dados de notificações de casos de DDA, no período entre 2011 e 2015 foram obtidos através do Sistema de Vigilância Epidemiológica (SIVEP- DDA-SP). O Indicador de Coleta e Tratamento de Água dos Municípios (ICTEM) foi obtido nos relatórios anuais da CETESB - Companhia Ambiental do Estado de São Paulo, (CETESB, 2018).

Para analisar aspectos sanitários dos municípios pertencentes ao GVE- VIII Mogi das Cruzes-SP, foram coletadas as seguintes informações junto a Secretaria Nacional de Saneamento Ambiental do Ministério das Cidades (SNIS, 2018): população atendida com água tratada (IN055), população atendida com esgoto (IN056), amostras fora do padrão coliformes (IN084_AE), população total e rural, essas informações são declaratórias e de competência dos municípios.

A fim de relacionar as informações obtidas de saneamento e informações municipais de notificações foi feita uma correlação de Pearson, usando a função estatística CORREL do programa Microsoft Excel 2010 (PINTO, 2011).

\section{RESULTADOS E DISCUSSÃO}

Quando do uso dos dados secundários provenientes da Secretaria Nacional de Saneamento Ambiental do Ministério das Cidades, foi observado que o município de Santa Isabel-SP (pertencente à GEV-VIII) não apresentava informações sobre o ano de 2013, por esse motivo esse município foi excluído desse estudo.

\section{Diagnóstico sanitário dos municípios do Grupo de Vigilância Epidemiológica - GVE- VIII Mogi das Cruzes.}

Na tabela 1 é apresentado um resumo das condições de cada município. Esses dados se referem às médias feitas a partir das informações do período, entre 2011 e 2015, das variáveis estudadas.

Os municípios com maior porcentagem de população rural: Biritiba Mirim, Guararema e Salesópolis, apresentaram menor atendimento quanto à coleta de esgoto e também com relação ao serviço de água tratada. Esses dados estão coerentes com as informações do documento Gasto público em Saneamento Basico -2014 (BRASIL, 2016), que aponta que na região sudeste enquanto $90 \%$ dos domicílios urbanos tem rede coletora de esgoto, apenas $15 \%$ dos domicílios rurais tem esse serviço. Esse mesmo documento indica que com relação ao acesso a rede de abastecimento de água a proporção entre urbano e rural é $96 \%$ para $25 \%$.

No caso da população urbana e rural atendida por coleta de esgoto (Figura 1), nenhum município oferece $100 \%$ de atendimento, e os municípios de Salesópolis, Biritiba Mirim e Guararema apresentaram os menores valores, sendo que os três últimos municípios com valores próximos ou inferiores a $50 \%$ na maior parte do período analisado. 
Ocorrência de casos de doenças diarreicas agudas e sua relação com os aspectos sanitários na região do Alto Tiete, São Paulo
Regina de Oliveira Moraes Arruda

Pedro Carmo de Souza

Edna Ferreira Rosini

Fernanda Dall'Ara Azevedo

Tabela 1 - Média dos resultados das condições sanitárias e notificações para cada município do Alto Tietê dos anos de 2011 a 2015.

\begin{tabular}{lcccccc}
\multicolumn{1}{c}{ Município } & IN055 & IN056 & IN084_AE & ICTEM & $\begin{array}{c}\% \text { pop. } \\
\text { rural }\end{array}$ & $\begin{array}{c}\% \\
\text { Notificações. }\end{array}$ \\
Arujá & 99,45 & 57,96 & 0,11 & 5,70 & 3,99 & 6,53 \\
\hline Biritiba Mirim & 56,34 & 48,85 & 0,45 & 7,21 & 14,17 & 5,24 \\
\hline Ferraz de Vasconcelos & 96,01 & 79,6 & 0,33 & 4,83 & 4,49 & 2,20 \\
\hline Guararema & 70,80 & 36,66 & 1,01 & 6,09 & 13,95 & 10,98 \\
\hline Guarulhos & 98,66 & 84,30 & 0,11 & 3,12 & 0,00 & 3,11 \\
\hline Itaquaquecetuba & 99,30 & 64,92 & 0,09 & 1,65 & 0,00 & 0,02 \\
\hline Mogi das Cruzes & 92,51 & 86,03 & 0 & 4,68 & 7,86 & 0,05 \\
\hline Poá & 99,83 & 96,73 & 0,31 & 8,93 & 1,58 & 0,77 \\
\hline Salesópolis & 64,54 & 54,52 & 0,24 & 8,03 & 36,35 & 11,81 \\
\hline Suzano & 99,41 & 86,53 & 0,26 & 5,98 & 3,52 & 3,02
\end{tabular}

Onde: IN055: \% população urbana e rural atendida com água tratada; IN056: \% população urbana e rural atendida com coleta de esgoto; IN084 AE: \% amostras fora do padrão coliformes; ICTEM: Índice de coleta e tratabilidade de esgoto. Fonte: Próprio autor.

Figura 1 - Porcentagem da população urbana e rural atendida por coleta de esgoto, dos municípios da VIII - Grupo de Vigilância Epidemiológica de Mogi das Cruzes.

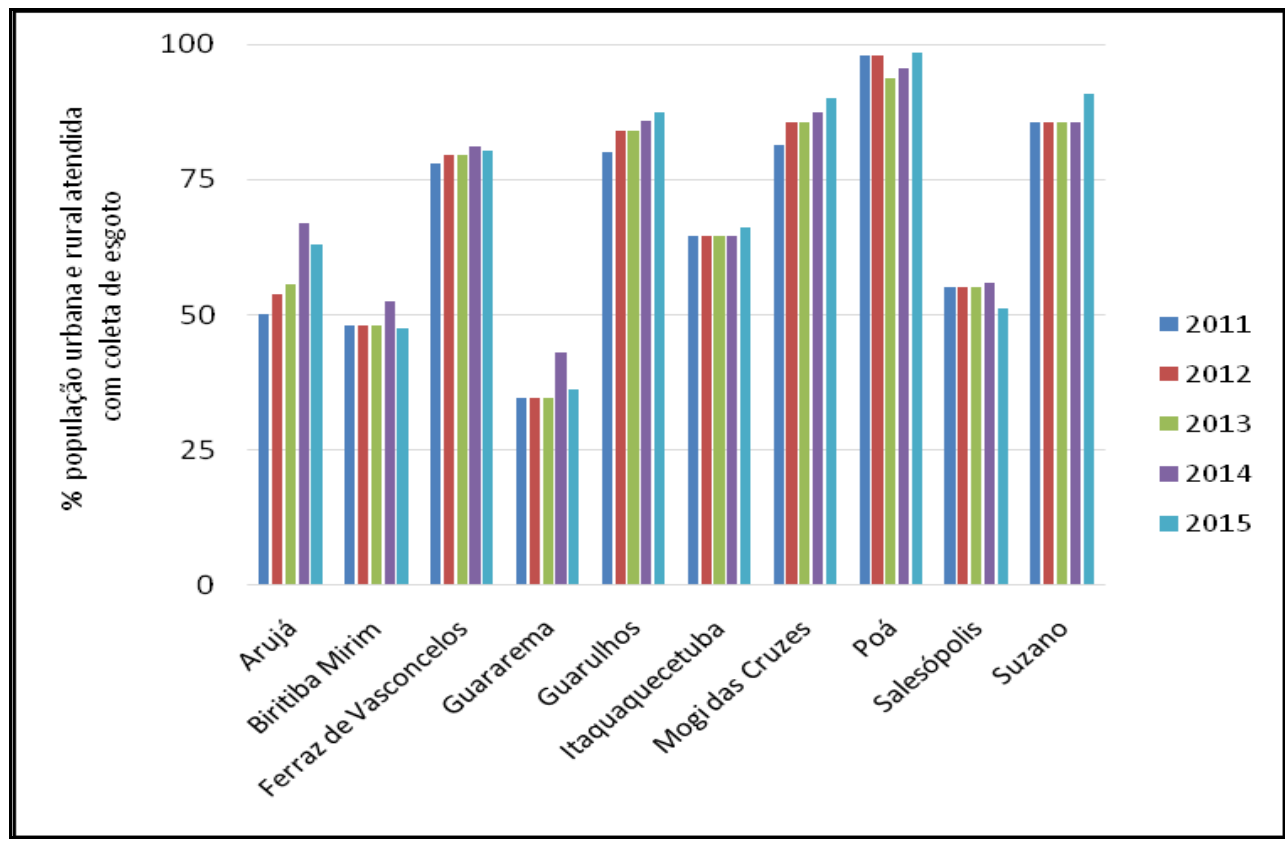

Fonte: SNIS, 2018 (adaptado).

Com relação a análise de potabilidade da água de abastecimento (BRASIL, 2012), a incidência das análises de coliformes totais fora do padrão para água de abastecimento ocorreu em todos os municípios, com exceção de Mogi das Cruzes. O município de Guararema foi o que apresentou mais inconformidades em relação à potabilidade de suas águas (Figura 2). 
Ocorrência de casos de doenças diarreicas agudas e sua relação com os aspectos sanitários na região do Alto Tiete, São Paulo
Regina de Oliveira Moraes Arruda Pedro Carmo de Souza

Edna Ferreira Rosini

Fernanda Dall'Ara Azevedo

Figura 2 - Incidência das análises de água de abastecimento com coliformes totais fora do padrão dos municípios da VIII - Grupo de Vigilância Epidemiológica de Mogi das Cruzes.

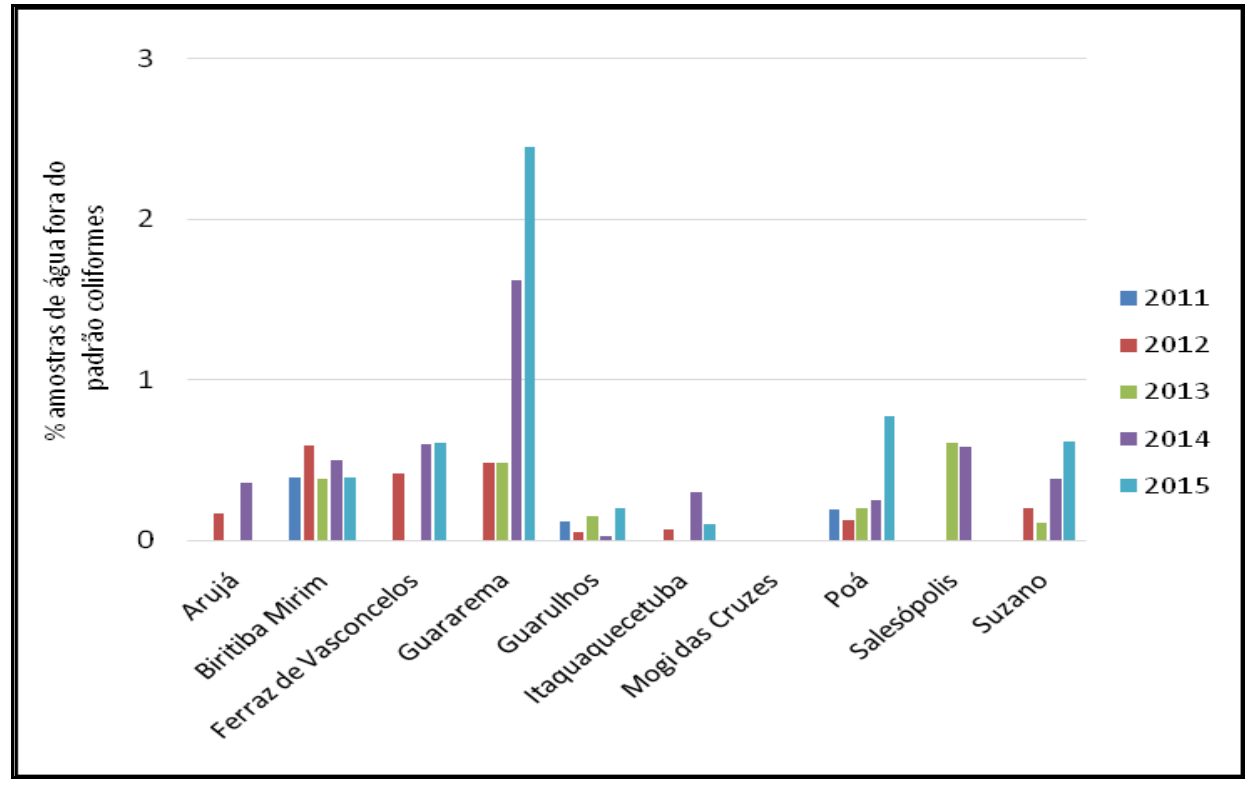

Fonte: SNIS, 2018 (adaptado).

Durante o período de estudo, 2011 a 2015, os maiores valores para o Indicador de Coleta e Tratabilidade de Esgoto de Município (ICTEM) foram apresentados pelos municípios de Biritiba-Mirim, Guararema, Poá e Salesópolis com melhora nesses serviços em 2014 e 2015, (Figura 3). Por outro lado, os menores valores deste indicador foram observados nos municípios de Itaquaquecetuba e Guarulhos, sendo que, em Guarulhos os valores do indicador caíram pela metade em 2014 e 2015 revelando piora nos serviços de coleta e tratabilidade de esgoto nos últimos dois anos de análise, corroborando os resultados apresentados por Gonçalves (2016).

Figura 3 - Evolução do Indicador de Coleta e Tratabilidade de Esgoto do Município (ICTEM), no período de 2011 a 2015, nos municípios de abrangência do GVE-VIII - Mogi das Cruzes.

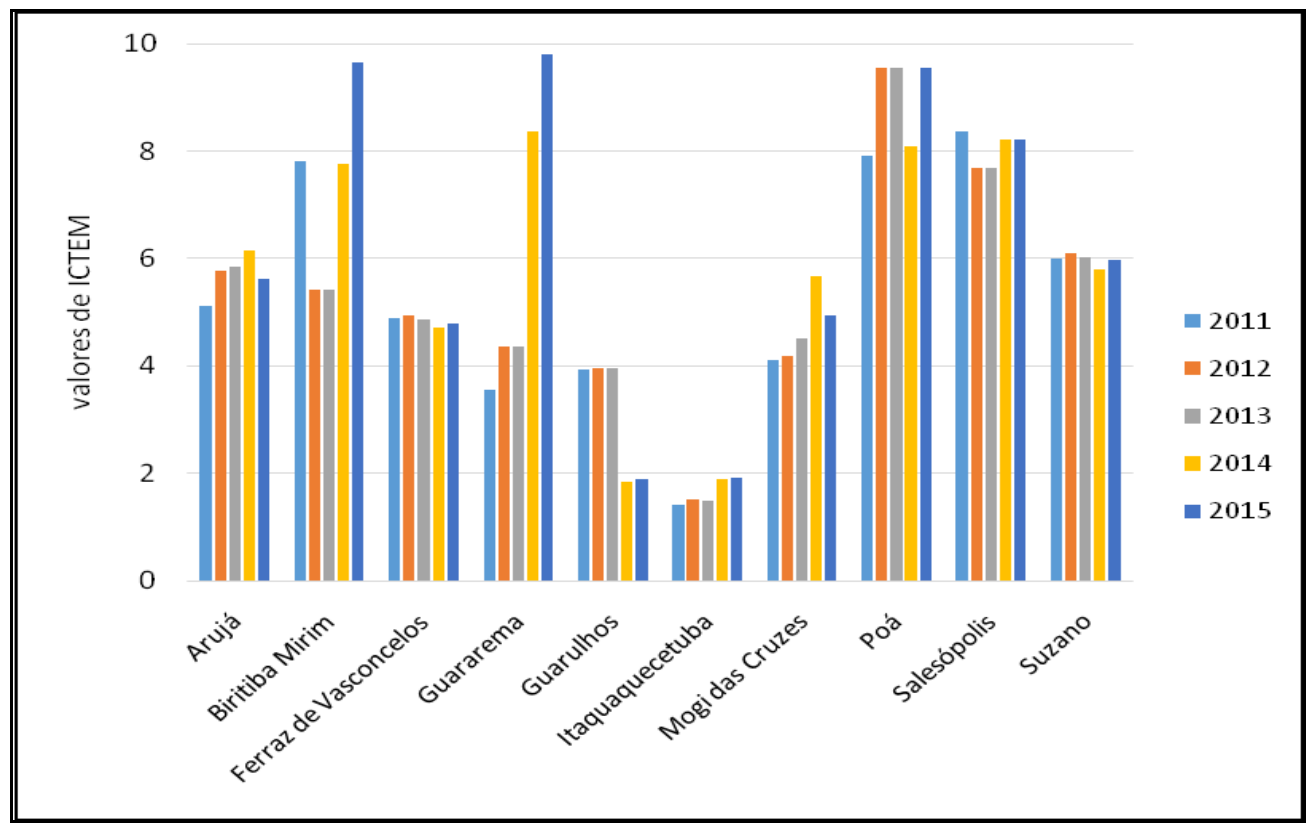

Fonte: CETESB, 2018 (adaptado) 
Ocorrência de casos de doenças diarreicas agudas e sua relação com os aspectos sanitários na região do Alto Tiete, São Paulo
Regina de Oliveira Moraes Arruda Pedro Carmo de Souza Edna Ferreira Rosini Fernanda Dall'Ara Azevedo

\section{Análise dos casos de Doenças diarreicas Agudas do GVE-VIII Mogi das Cruzes}

Os dados de notificações de casos de DDA obtidos pelo Sistema de Vigilância Epidemiológica (SIVEP- DDA - SP) nos anos de 2011 a 2015, do Grupo de Vigilância Epidemiológica - GVE- VIII Mogi das Cruzes, estão apresentados na figura 4.

De acordo com os documentos pesquisados, notou-se a ausência expressiva de notificações em alguns períodos, o que indica a subnotificação da ocorrência de casos de DDA. Esse fato também foi observado por Buffon, Mendonça (2017) que a partir das subnotificações das DDA's não pôde ser realizada uma análise mais detalhada, tanto da proporção de influência dos elementos climáticos como das transformações socioambientais urbanas em Curitiba e seus reflexos diretos sobre a doença.

Os municípios de Biritiba Mirim, Guararema e Salesópolis apresentaram os maiores valores de notificações e também estão entre os municípios que apresentaram os piores resultados na pesquisa realizada com parâmetros relacionados ao saneamento. Por outro lado, Guarulhos e Itaquaquecetuba, também entre os municípios com os piores resultados em relação ao saneamento básico e outros municípios como Ferraz de Vasconcelos, Mogi das Cruzes, Poá e Suzano apresentaram números de notificação de DDA baixos. Este resultado se deve, provavelmente, a subnotificação da ocorrência de casos de DDA, pois Itaquaquecetuba, Mogi das Cruzes e, especialmente, Guarulhos com aproximadamente 1.300 milhões de habitantes são municípios com grandes populações, onde comumente ocorre a distribuição irregular de renda gerando grandes bolsões de pobreza que frequentemente expõe a população a condições sanitárias precárias que contribuem com o aumento de DDA's (OLIVEIRA et al.,2009).

Figura 4 - Evolução dos casos de DDA no período de 2011 a 2015 nos municípios de abrangência do GVE-VIII - Mogi das Cruzes

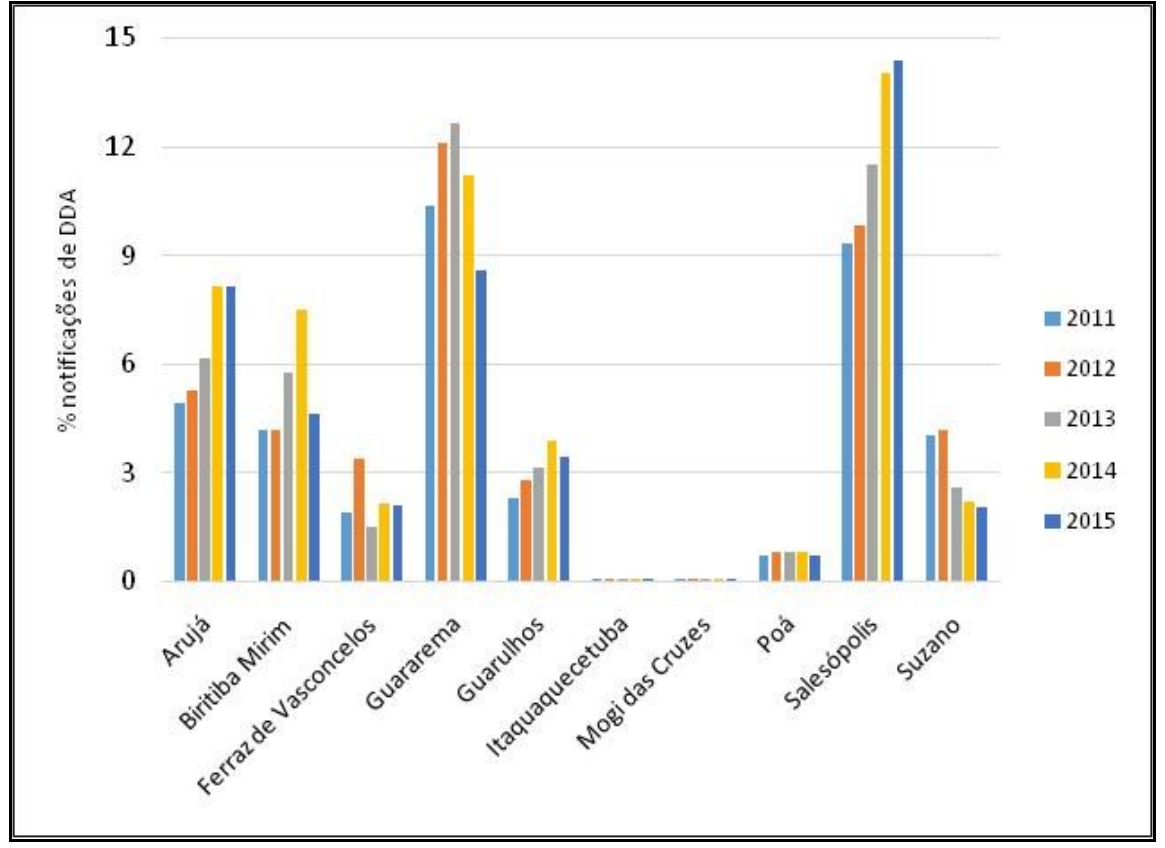

Fonte: SIVEP- DDA (adaptado)

Oliveira et al. (2020) também apontam que as DDA's estão atreladas a fatores socioeconômicos, culturais, e principalmente ambientais e de infraestrutura, e nesta pesquisa os municípios com menores taxas de atendimento de rede de esgoto apresentam o maior número de notificações.

Para a análise dos dados utilizou-se a correlação (Pinto, 2011) apresentada na Tabela 2, valores superiores a 0,70 são considerados como fortemente relacionados, entre 0,40 e 0,69 são moderadamente relacionados e entre 0,20 e 0,39 temos os considerados de fraca relação. 
Observou-se uma forte correlação entre água tratada e coleta de esgoto (Tabela 2), e principalmente a relação entre a diminuição de notificações de doenças diarreicas com o aumento da população atendida com água tratada e com coleta de esgoto. Sendo assim, os dados do presente estudo são corroborados por Siqueira et al. (2017) que afirmam que as doenças relacionadas ao saneamento ambiental inadequado (DRSAI) se manifestam principalmente sob a forma de diarreias, e estão entre as principais causas de morbimortalidade nos países de economias em desenvolvimento. Sabe-se que a prevalência dessas enfermidades constitui indicativo de um sistema de saneamento frágil e deficiente

Nesta pesquisa não se encontrou relação entre os casos de notificação de doenças diarreicas e o ICTEM - Índice de coleta e tratabilidade de esgoto nos municípios. Malheiros et al. (2017) trabalhando com indicador de internações por Doenças Relacionadas ao Saneamento Ambiental (DRSA) inadequado e ICTEM também chegaram a coeficientes de correlação muito fracos, reforçando a teoria da multicausalidade (conjunto de fatores determinantes) na relação qualidade ambiental e saúde pública

Observou-se uma correlação entre porcentagem de população rural e casos de notificação. Provavelmente esse fato ocorreu devido à dificuldade para que serviços como abastecimento de água e coleta de esgotos cheguem à zona rural (OLIVEIRA et al., 2009; MOTA et al., 2015).

Bühler et al. (2014), em estudo de epidemiologia ambiental sobre morbimortalidade por diarreia infantil no Brasil com vários indicadores, também concordam que os relacionados ao saneamento básico inadequado distinguiram-se dos demais. $\mathrm{E}$ concordam que as políticas públicas socioambientais devem priorizar o saneamento básico enquanto medidas de prevenção e proteção à saúde infantil, particularmente nas regiões com piores condições socioeconômicas do país.

Tabela 2 - Correlação de Pearson aplicada aos parâmetros analisados.

\begin{tabular}{crrrrrc}
\hline & IN055 & IN056 & IN084_AE & ICTEM & \% pop rural & Notificações \\
\hline IN055 & 1,00 & & & & & \\
\hline IN056 & 0,73 & 1,00 & & & & \\
\hline IN084 & $-0,31$ & $-0,33$ & 1,00 & & & \\
\hline ICTEM & $-0,39$ & 0,06 & 0,42 & 1,00 & & \\
\hline \% pop rural & $-0,79$ & $-0,54$ & 0,17 & 0,46 & 1,00 & \\
\hline Notificações & $-0,66$ & $-0,73$ & 0,32 & 0,35 & 0,75 & 1,00 \\
\hline
\end{tabular}

Onde: IN055 (\%população atendida com água tratada); N056 (\%população atendida com esgoto); IN084_AE (\% amostras de água fora do padrão coliformes); ICTEM (Indicador de Coleta e Tratabilidade de Esgoto do Município); \% população rural no município e notificações (\% de casos de notificações de doenças diarreicas agudas). Fonte: Próprio autor.

Uhr, Schmechel, Uhr (2016) verificaram que a redução da morbidade é mais significativa quando há um aumento dos domicílios ligados a rede coletora de esgotos e com coleta adequada de resíduos sólidos, quando comparada aos domicílios com canalização interna de água. Os autores também afirmam que o gasto público com tratamento hospitalar pode ser reduzido apenas ampliando-se a cobertura dos serviços básicos de saneamento.

\section{CONCLUSÕES}

A partir desta pesquisa, realizada na região do Alto Tietê-SP conseguiu-se relacionar o número de casos de notificação de DDA's com população não atendida por água tratada e população não atendida por coleta de esgoto, indicando a importância do saneamento para a saúde da população estudada. 
Os municípios com maior porcentagem de população rural também apresentaram um maior número de notificações de DDA's isso pode ser explicado pela dificuldade de oferta de serviços sanitários em áreas rurais.

A escolha do Grupo de Vigilância Epidemiológica - GVE-VIII de Mogi das Cruzes, como área de estudo, foi assertiva por ter municípios bastante diversificados quanto a aspectos sanitários, população rural e casos de notificações de DDA'S.

\section{REFERENCIAS}

BRAGA, B. Introdução à Engenharia Ambiental: Conhecimento especializado - Saneamento e Tecnologia Ambiental. $2^{\mathrm{a}}$ ed. São Paulo: Prentece Hall, 2003.

BRASIL. Ministério da Saúde. Grupo de vigilância epidemiológica - GVE. 2005. Disponível em: http://www.saude.sp.gov.br/cve-centro-de-vigilancia-epidemiologica-prof.-alexandrevranjac/institucional/sobre-o-cve. Acesso em: 14 out 2017.

BRASIL. Ministério da Saúde. PORTARIA №- 2.914, DE 12 DE DEZEMBRO DE 2011. Dispõe sobre os procedimentos de controle e de vigilância da qualidade da água para consumo humano e seu padrão de potabilidade. Brasília. 2012.

BRASIL. Ministério da Saúde. Secretaria de Vigilância em Saúde. Departamento de Vigilância de Doenças e Agravos não Transmissíveis e Promoção da Saúde. Saúde Brasil 2017: uma análise da situação de saúde e os desafios para o alcance dos objetivos de desenvolvimento sustentável. - Brasília: Ministério da Saúde, 2018.

BRASIL. Ministério das Cidades. Gasto Público em Saneamento Básico - 2014. Brasília, Secretaria Nacional de Saneamento Ambiental - SNSA. Ministério das Cidades, 2016.

BUFFON, E.A.M.; MENDONÇA, F.A. Análise integrada da vulnerabilidade à diarreia aguda em Curitiba, Confins. Revue franco-brésilienne de géographie/Revista franco-brasilera de geografia, n. 33, 2017. Disponível em: http://journals.openedition.org/confins/12610 Acesso em: 17 out 2018. https://doi.org/10.4000/confins. 12610

BÜHLER, H.F.; IGNOTTI, E.; NEVES, S.M.A.D.S.; HACON, S.D.S. Análise espacial de indicadores integrados de saúde e ambiente para morbimortalidade por diarreia infantil no Brasil, 2010. Cadernos de Saúde Pública, v. 30, p. 1921-1934, 2014. Disponível em: https://www.scielosp.org/pdf/csp/2014.v30n9/1921-1934/pt 23 JUL 2019 Acesso: 17 out 2018. https://doi.org/10.1590/0102-311X00078013

CETESB - Companhia Ambiental do Estado de São Paulo. Apêndice D-Índices de Qualidade das Águas. 2018. Disponível em: https://cetesb.sp.gov.br/aguas-interiores/publicacoes-e-relatorios/ Acesso em: 17 out 2018.

GONÇALVES, J.J.S. A contribuição do município de Guarulhos para a qualidade da água do rio Baquirivu-Guaçu. Dissertação em Análise Geoambiental. Universidade Guarulhos. 2016. Disponível em: http://hdl.handle.net/123456789/667 Acesso em: 25 set 2017.

JACOBI, P.R. Moradores e meio ambiente na cidade de São Paulo. Previd. dados, v. 10, n. 1, p. 2650, $1995 \quad$ Disponível em: http://www.scielo.br/scielo.php?script=sci_nlinks\&ref=000128\&pid=S01023098200600010000400007 \&lng=pt Acesso em: 17 de fev de 2017.

MALHEIROS, T.F.; MALHEIROS, A.A.; DE MELO, T.P.; RAMOS, M.C.G.; DE FREITAS, G.N. Um estudo sobre o indicador de doenças relacionadas ao saneamento ambiental inadequado - DRSAI aplicado ao contexto do saneamento básico nas Bacias do Piracicaba-Capivari, Jundiaí-PCJ. In: Congresso ABES/FENASAN-2017, Congresso Nacional de Meio Ambiente e Saneamento 2017. Anais...São Paulo, SP. 2017. Disponível em: https://www.saneamentobasico.com.br/wpcontent/uploads/2018/08/VII-027.pdf Acesso em: 10 jun 2019.

MIRANDA, G.M.; BRAGA, R. Indicadores de desempenho na gestão dos recursos hídricos nos municípios das bacias hidrográficas dos rios Piracicaba, Capivari e Jundiaí. Revista GeoNordeste, $n$. 
2, 2011. Disponível em: https://seer.ufs.br/index.php/geonordeste/article/view/2417 Acesso em: 05 jul 2019.

MOTA, J.J.P.; SOUSA, C.S.S.; DA SILVA, A.C. Saneamento básico e seu reflexo nas condições socioambientais da zona rural do Baixo Munim (Maranhão). Caminhos de Geografia, v. 16, n. 54, 2015. Disponível em: http://www.seer.ufu.br/index.php/caminhosdegeografia/article/view/26850 Acesso em: 10 jun $2019 .$.

OLIVEIRA, D.S.C.; GUIMARÃES, M.J.B.; MEDEIROS, Z. Modelo produtivo para Leptospirose. Revista de Patologia Tropical. v. 38, n.1, p.17-26, jan-mar, 2009. Disponível em: https://www.revistas.ufg.br/iptsp/article/view/6205 Acesso em: 15 dez 2018. https://doi.org/10.5216/rpt.v38i1.6205

OLIVEIRA, H.M.; GOMES, K.R.B.; FERREIRA, C.R.S.; FERREIRA, C.R.S.; NASCIMENTO, V.B.; DIAS, W. W. D. S. Doença diarreica aguda em menores de 5 anos em um hospital da fronteira do Brasil. Revista científica del Amazonas, v. 3, n. 5, p. 30-40, 2020. Disponível em: https://www.revistadelamazonas.info/index.php/amazonas/article/view/28 Acesso em: 15 de fev 2020.

PAZ, M.G.A.; ALMEIDA, M.F.; GÜNTHER, W.M.R. Prevalência de diarreia em crianças e condições de saneamento e moradia em áreas periurbana de Guarulhos, SP. Revista Brás Epidemiol, São Paulo, v. 15, p. 188-197, 2012. Disponível em: https://www.scielosp.org/scielo.php?pid=S1415790X2012000100017\&script=sci_arttext\&tIng=es Acesso em: 10 jun 2019 . https://doi.org/10.1590/S1415-790X2012000100017

PINTO, M.P. Microsoft Excel 2010. $1^{\mathrm{a}}$ edição. Famalicão, Portugal: Ediçoes Centro Atlântico, 2011. http://centroatl.pt/titulos/so/capas-pdfs/excerto-livro-ca-excel2010.pdf acesso em 15 de fev 2020.

QUEIROZ, J.T.M.; HELLER,' L; SILVA, S.R. Análise da correlação de ocorrência da doença diarreica aguda com a qualidade da água para consumo humano no município de Vitória - ES. Saúde e Sociedade, v. 18, p. 479-489, 2009. Disponível em: https://www.scielosp.org/article/sausoc/2009.v18n3/479-489/pt/ Acesso em: 25 jun 2019. https://doi.org/10.1590/S0104-12902009000300012

SIQUEIRA, M.S.; ROSA, R.D.S.; BORDIN, R.; NUGEM, R.D.C. Internações por doenças relacionadas ao saneamento ambiental inadequado na rede pública de saúde da região metropolitana de Porto Alegre, Rio Grande do Sul, 2010-2014. Epidemiologia e Serviços de Saúde. 2017, v. 26, n. 4, pp. 795-806. https://doi.org/10.5123/S1679-49742017000400011

SNIS -Sistema Nacional de Informações sobre Saneamento. Ministério do Desenvolvimento Regional. Secretaria Nacional de Saneamento. Série histórica. 2018. Disponível em: http://app3.cidades.gov.br/serieHistorica/\# Acesso em: 10 dez 2018.

UHR, J.G.Z., SCHMECHEL, M.; UHR, D.D.A.P. Relação entre saneamento básico no Brasil e saúde da população sob a ótica das internações hospitalares por doenças de veiculação hídrica. Revista de Administração, Contabilidade e Economia da Fundace, v. 7, n. 2, 2016. https://www.fundace.org.br/revistaracef/index.php/racef/article/view/104/pdf_19 Acesso em: 10 jun 2019. https://doi.org/10.13059/racef.v7i2.104

WHO. World Health Organization. The treatment of diarrhea: a manual for physicians and other senior health workers. Geneva: WHO; 2005. Disponível em: http://whqlibdoc.who.int/publications/2005/9241593180.pdf Acesso em: 24 mai 2012. 